\title{
Social factors and hospitalization
}

\section{Les facteurs sociaux et I'hospitalisation}

In the present issue of the Canadian Respiratory Journal, a group from St Paul's Hospital in Vancouver (British Columbia) examined determinants of length of hospital stay and frequency of readmissions in patients with acute exacerbations of chronic obstructive pulmonary disease (COPD) (1). The usual suspects emerged as important, including disease severity and the presence of comorbidities $(2,3)$. They also looked at the influence of social factors as determinants of length of stay and readmission. The study had limitations in that it was retrospective and limited to data available in routine patient charts, and studied the St

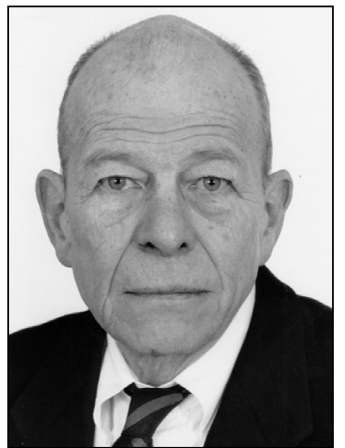

Nick R Anthonisen ans le présent numéro de la Revue canadienne Ue pneumologie, un groupe du St. Paul Hospital de Vancouver (en Colombie-Britannique) a examiné les déterminants de la durée d'hospitalisation et de la fréquence des réhospitalisations chez les patients présentant des exacerbations aiguës de la maladie obstructive pulmonaire chronique (MOPC) (1). Les suspects habituels étaient importants, y compris la gravité de la maladie et la présence de comorbidités (2,3). Le groupe a également examiné l'influence des facteurs sociaux comme déterminants de la durée d'hospitalisation et des réhospitalisations. L'étude comportait des Paul's population, which is probably less heterogeneous than that of other big-city hospitals. Nevertheless, they found that the presence of a spouse reduced readmission rates and that intervention by social workers was a marker of increased length of stay.

These results are not surprising, and probably apply to hospitalization due to virtually any cause. The decision to admit, or to not discharge any patient is, and should be, influenced by the care available to the patient outside the hospital. Patients who do not have spouses have to care for themselves, and are likely to receive worse care than those with an involved, interested other party such as a spouse. Social workers are usually consulted when it is not clear whether a hospitalized patient will be able to take care of him- or herself, and alternate plans have to be considered, a process that can take considerable time, lengthening the hospital stay. Indeed, contemporary studies of length of stay of hospitalized patients with COPD frequently censor data after seven to 10 days, on the assumption that longer stays are more related to social conditions than the actual disease. Although Wong et al (1) do not say so, I am virtually certain that social worker consultations were much more common in patients without an available spouse than in those with a spouse. These are only some of the social factors affecting hospitalization. Wong et al (1) adjusted their data to account for age, but age may be regarded as a social factor as well as a biological feature; for example, as people get older, their chances of having a spouse decreases. They apparently could not assess their patients' socioeconomic status, although this can be done (roughly) in Manitoba. However, had they done so, I am sure that low socioeconomic status would have increased readmissions and prolonged hospital stay; it certainly increases COPD mortality (4).

Nearly fifty years ago, as part of my medical residency program, I served in the infirmary of a large university, examining and treating sick students. The patients were, of course, largely young and healthy, and most of the diseases seen were selflimited, although I can remember one episode of appendicitis and a major gastrointestinal bleed. Although most illnesses were trivial, the infirmary had beds and followed a very liberal admissions policy; if there was any doubt about a student, we limites, car elle était rétrospective, était restreinte aux données disponibles dans le dossier des patients et à la population de l'hôpital, probablement moins hétérogène que celles d'autres hôpitaux des grands centres. Néanmoins, le groupe a découvert que la présence d'un conjoint diminuait le taux de réhospitalisations et que l'intervention de travailleurs sociaux constituait un marqueur d'hospitalisation plus prolongée.

Ces résultats ne sont pas surprenants et s'appliquent probablement aux hospitalisations pour à peu près toutes les causes. La décision d'hospitaliser ou de ne pas donner son congé à un patient est et devrait dépendre des soins offerts au patient à l'extérieur de l'hôpital. Les patients qui n'ont pas de conjoint doivent se soigner eux-mêmes et risquent de recevoir de moins bons soins que ceux qui habitent avec une personne intéressée et engagée comme un conjoint. D'ordinaire, les travailleurs sociaux sont consultés lorsqu'on ne sait pas si un patient hospitalisé pourra s'occuper de lui-même et qu'il faut envisager d'autres dispositions, un processus qui peut être très long et rallonger l'hospitalisation. D'ailleurs, les études contemporaines sur la durée d'hospitalisation des patients atteints d'une MOPC et hospitalisés recensent souvent les données après sept à dix jours, selon l'hypothèse que les hospitalisations plus longues sont davantage reliées aux conditions sociales qu'à la maladie même. Même si Wong et coll. (1) ne le disent pas, je suis à peu près certain que les consultations des travailleurs sociaux étaient beaucoup plus courantes chez les patients qui n'avaient pas de conjoint disponible que chez ceux qui en avaient un. Ce ne sont là que quelques-uns des facteurs qui influent sur l'hospitalisation. Wong et coll. (1) ont rajusté leurs données pour tenir compte de l'âge, mais l'âge peut constituer un facteur social autant qu'une caractéristique biologique. Par exemple, à mesure que les gens vieillissent, leur chance d'avoir un conjoint diminue. Apparemment, ils ne pouvaient pas évaluer le statut socio-économique de leurs patients, même si c'est (plus ou moins) possible au Manitoba. Cependant, s'ils l'avaient fait, je suis sûr qu'un statut socio-économique faible aurait accru les réhospitalisations et les hospitalisations prolongées. En tout cas, il accroît la mortalité de la MPOC (4).

Il y a près de cinquante ans, dans le cadre de ma résidence en médecine, j'ai travaillé à l'infirmerie d'un grand hôpital, 
were instructed to put him or her to bed in our facility. This was very sensible. Students could not be trusted to take care of themselves, and in their dormitories there was no one else around to do it. The social context was the chief determinant of admissions policy. I would argue that this applies to big-city emergency rooms as well, and that this is appropriate.

The above being said, it is important to remember that there is little that happens to COPD patients in either emergency rooms or on hospital wards that cannot be duplicated in the home, and that if appropriate therapy is introduced early in a COPD exacerbation, these episodes can frequently be avoided (5). Emergency room or hospital admission represents a failure of outpatient management, which also has social determinants.

Nick R Anthonisen MD Editor-in-Chief, Canadian Respiratory Journal

\section{REFERENCES}

1. Wong AWM, Gan WQ, Burns J, Sin DD, van Eeden SF. Acute exacerbation of chronic obstructive pulmonary disease: Influence of social factors in determining length of hospital stay and readmission rates. Can Respir J 2008;15:361-364.

2. Gudmundsson G, Gislason T, Janson C, et al. Risk factors for rehospitalisation in COPD: Role of health status, anxiety and depression. Eur Respir J 2005;26:414-9.

3. Osman IM, Godden DJ, Friend JA, Legge JS, Douglas JG. Quality of life and hospital re-admission in patients with chronic obstructive pulmonary disease. Thorax 1997;52:67-71.

4. Prescott E, Godtfredsen N, Vestbo J, Osler M. Social position and mortality from respiratory diseases in males and females. Eur Respir J 2003;21:821-6.

5. Bourbeau J, Nault D. Self-management strategies in chronic obstructive pulmonary disease. Clin Chest Med 2007;28:617-28. examinant et traitant les étudiants malades. Bien sûr, la plupart des patients étaient jeunes et en santé, et la plupart des maladies étaient spontanément résolutives, même si je me rappelle un cas d'appendicite et une grave hémorragie gastrointestinale. La plupart des maladies étaient bénignes, mais l'infirmerie comportait des lits et respectait une politique d'hospitalisation très libérale. S'il y avait un doute quelconque au sujet d'un étudiant, nous devions l'installer dans un lit. C'était très sensé. On ne pouvait pas s'attendre que les étudiants s'occupent d'eux-mêmes, et personne ne pouvait le faire aux résidences. Le contexte social était le principal déterminant de la politique d'hospitalisation. Selon moi, ce principe s'applique aussi aux urgences des grandes villes, et c'est judicieux.

Cela étant dit, il faut se souvenir qu'auprès des patients atteints d'une $\mathrm{MOPC}$, on peut reproduire à domicile à peu près tout ce qui se passe à l'urgence ou dans un service hospitalier et que si un traitement convenable est adopté assez tôt lors d'une exacerbation, il est souvent possible d'éviter l'hospitalisation (5). L'urgence ou l'hospitalisation représente un échec de la prise en charge des consultations externes, qui comporte aussi des déterminants sociaux.

Nick R Anthonisen MD

Rédacteur en chef, Revue canadienne de pneumologie

\section{RÉFÉRENCES}

1. Wong AWM, Gan WQ, Burns J, Sin DD, van Eeden SF. Acute exacerbation of chronic obstructive pulmonary disease: Influence of social factors in determining length of hospital stay and readmission rates. Can Respir J 2008;15:361-364.

2. Gudmundsson G, Gislason T, Janson C et coll. Risk factors for rehospitalisation in COPD: Role of health status, anxiety and depression. Eur Respir J 2005;26:414-9.

3. Osman IM, Godden DJ, Friend JA, Legge JS, Douglas JG. Quality of life and hospital re-admission in patients with chronic obstructive pulmonary disease. Thorax 1997;52:67-71.

4. Prescott E, Godtfredsen N, Vestbo J, Osler M. Social position and mortality from respiratory diseases in males and females. Eur Respir J 2003;21:821-6.

5. Bourbeau J, Nault D. Self-management strategies in chronic obstructive pulmonary disease. Clin Chest Med 2007;28:617-28. 


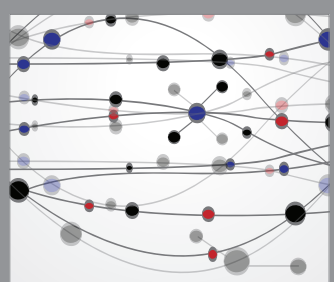

The Scientific World Journal
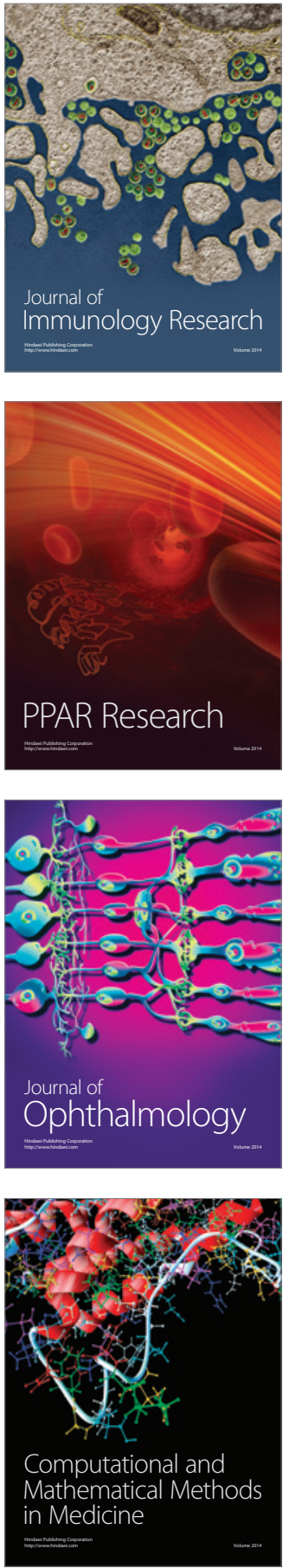

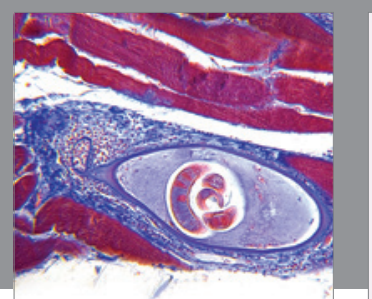

Gastroenterology Research and Practice

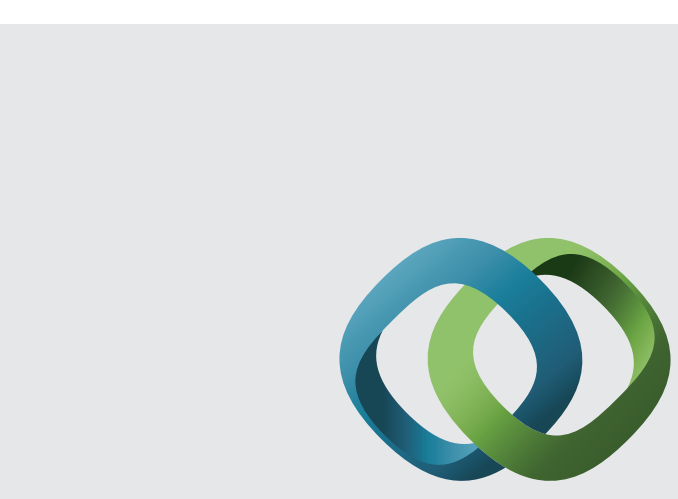

\section{Hindawi}

Submit your manuscripts at

http://www.hindawi.com
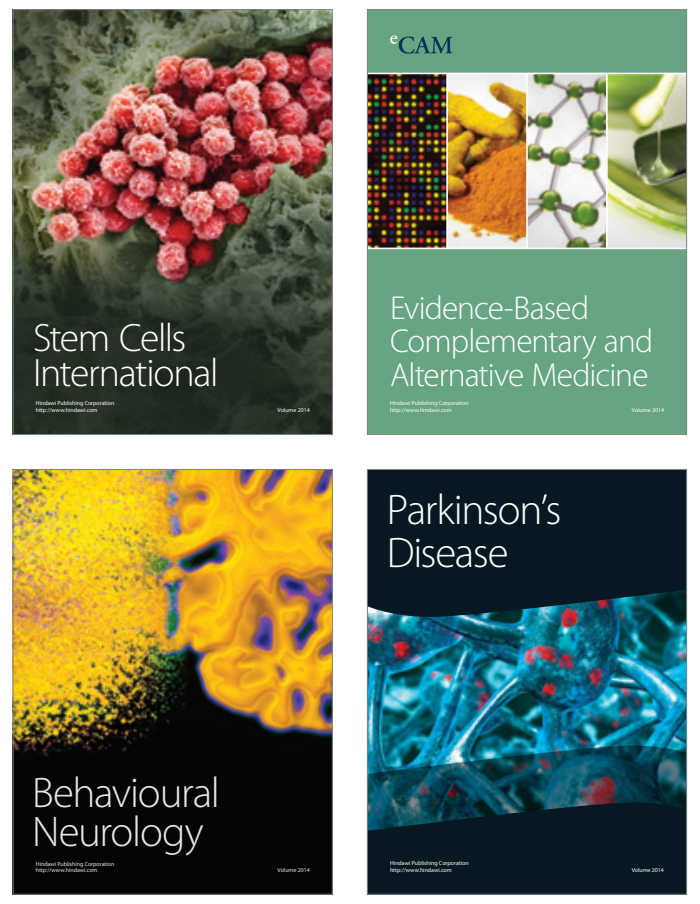
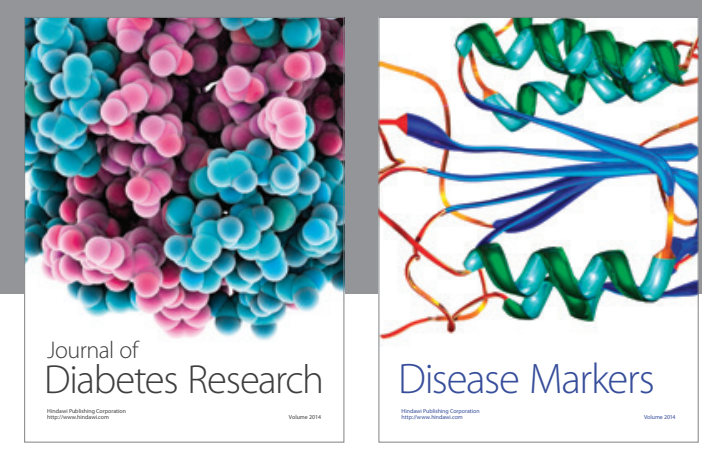

Disease Markers
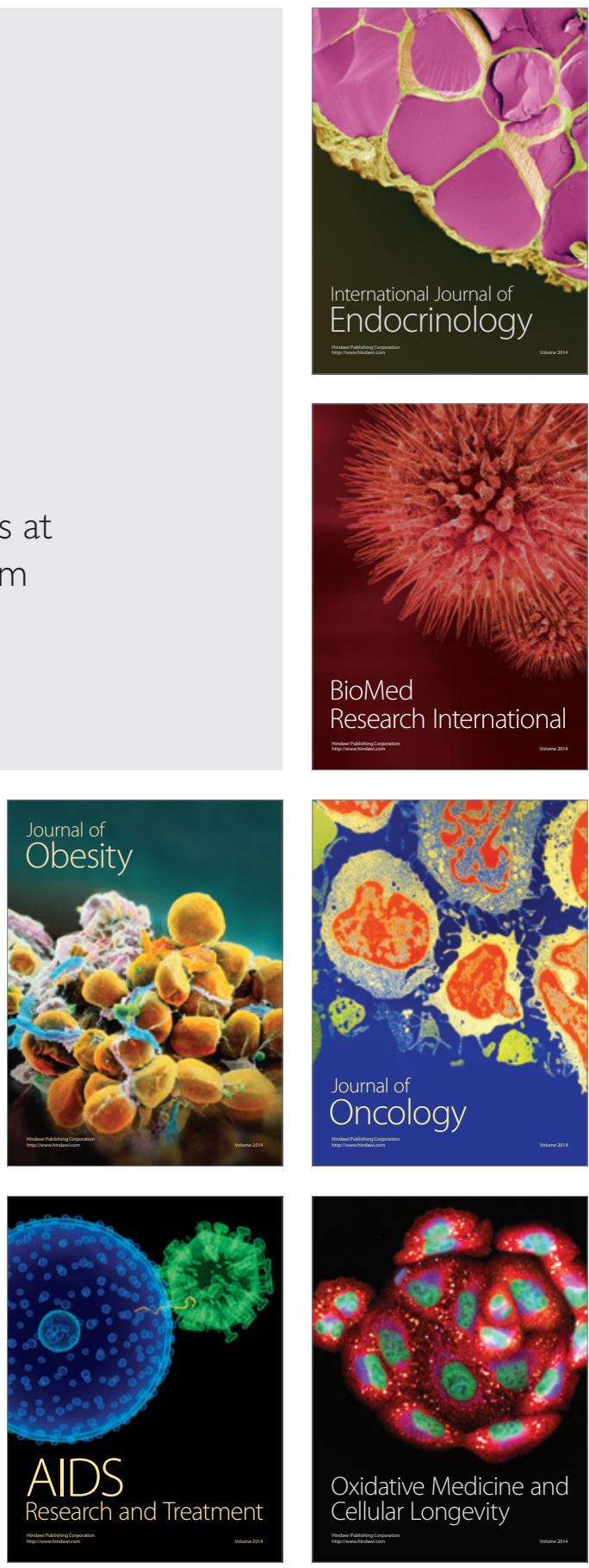